\title{
On the powers of Voiculescu's circular element
}

\author{
by \\ Ferenc Oravecz (Budapest)
}

\begin{abstract}
The main result of the paper is that for a circular element $c$ in a $C^{*}$ probability space, $\left(c^{n}, c^{n^{*}}\right)$ is an $R$-diagonal pair in the sense of Nica and Speicher for every $n=1,2, \ldots$ The coefficients of the $R$-series are found to be the generalized Catalan numbers of parameter $n-1$.
\end{abstract}

0. Introduction. Circular elements of a $C^{*}$-probability space play a central role in Voiculescu's free probability theory, and they provide one of the most frequently used $*$-distributions (see e.g. [11]). An important characterization of non-commutative random variables is based on the $R$-series of certain pairs. The $R$-series of a pair is an infinite power series in two non-commuting indeterminates, and can be taken as the free analogue of the Fourier transform [6]. Now an element $c$ is circular if the $R$-series of the pair $\left(c, c^{*}\right)$ is of the form

$$
R_{\left(c, c^{*}\right)}\left(z_{1}, z_{2}\right)=z_{1} z_{2}+z_{2} z_{1}
$$

As a natural extension of the notion of circular element, we will call a pair $\left(a_{1}, a_{2}\right)$ of non-commutative random variables a circular pair if its $R$-series is

$$
R_{\left(a_{1}, a_{2}\right)}\left(z_{1}, z_{2}\right)=z_{1} z_{2}+z_{2} z_{1} .
$$

In [7] Nica and Speicher introduced the notion of $R$-diagonal pairs in the following way. The pair $(x, y)$ of non-commutative random variables is $R$-diagonal if the $R$-series of $(x, y)$ is of the form

$$
R_{(x, y)}\left(z_{1}, z_{2}\right)=\sum_{k=1}^{\infty} \alpha_{k}\left(z_{1} z_{2}\right)^{k}+\sum_{k=1}^{\infty} \alpha_{k}\left(z_{2} z_{1}\right)^{k}
$$

for some sequence $\left\{\alpha_{k}\right\}_{k=1}^{\infty}$ of complex coefficients. The aim of this definition was to establish a class of pairs of non-commutative random variables which contains the pair $\left(c, c^{*}\right)$ obtained from a circular element $c$, and $\left(u, u^{*}\right)$

2000 Mathematics Subject Classification: 46L53, 06A07.

Supported by OTKA T023447. 
obtained from a Haar unitary element $u$. Haar unitary elements form another important type of non-commutative random variables [10], characterized by the $R$-series of the pair $\left(u, u^{*}\right)$ having the form

$$
R_{\left(u, u^{*}\right)}\left(z_{1}, z_{2}\right)=\sum_{k=1}^{\infty}(-1)^{k-1} c_{k-1}\left(z_{1} z_{2}\right)^{k}+\sum_{k=1}^{\infty}(-1)^{k-1} c_{k-1}\left(z_{2} z_{1}\right)^{k},
$$

where $c_{k}=\frac{1}{k+1}\left(\begin{array}{c}2 k \\ k\end{array}\right)$ is the $k$ th Catalan number [3].

In [8] and [2] various other conditions equivalent to $R$-diagonality are given.

The main result of this paper (stated as Theorem 3.2) is that for any circular pair $\left(b_{1}, b_{2}\right)$, the pair $\left(b_{1}^{n}, b_{2}^{n}\right)$ is $R$-diagonal for every $n \geq 1$. The sequence $\left\{\alpha_{k}\right\}_{k=1}^{\infty}$ of coefficients of the $R$-series of $\left(b_{1}^{n}, b_{2}^{n}\right)$ is found to be $\left\{{ }_{n-1} c_{k}\right\}_{k=1}^{\infty}$, the generalized Catalan numbers of parameter $n-1$ (see [3]), given by the formula

$$
{ }_{n-1} c_{k}=\frac{1}{(n-2) k+1}\left(\begin{array}{c}
(n-1) k \\
k
\end{array}\right) \quad(k=0,1, \ldots ; n=2,3, \ldots) .
$$

The structure of the paper is the following. In Section 1 we review the necessary elements of non-commutative probability theory. In Section 2 we state and prove some combinatorial lemmas concerning rather special types of partitions of certain sets. These lemmas are used for the proof of our main result, Theorem 3.2, which can be found in Section 3, along with some remarks and consequences.

Soon after this paper was completed, F. Larsen obtained some more general results on powers of $R$-diagonal pairs. These results can be found in $[5]$.

1. Preliminaries. In this section we briefly review some basic elements of non-commutative probability theory used throughout the paper. For further details see e.g. $[2,12]$ and the references listed there.

If $\mathcal{A}$ is a unital algebra over $\mathbb{C}$ and $\varphi: \mathcal{A} \rightarrow \mathbb{C}$ is a linear functional normalized by $\varphi(\mathbf{1})=1$, then we call the pair $(\mathcal{A}, \varphi)$ a non-commutative probability space. In this case the elements of $\mathcal{A}$ are called non-commutative random variables and for $a \in \mathcal{A}, \varphi\left(a^{n}\right)$ is called the $n$th moment of $a$. If we require in addition that $\mathcal{A}$ is a $C^{*}$-algebra and $\varphi$ is positive, then $(\mathcal{A}, \varphi)$ is called a $C^{*}$-probability space.

In the rest of this section $(\mathcal{A}, \varphi)$ stands for a non-commutative probability space.

We denote the set of all infinite power series in the non-commuting indeterminates $X_{1}, \ldots, X_{k}$ by $C_{\infty}\left\langle X_{1}, \ldots, X_{k}\right\rangle$. For $F \in C_{\infty}\left\langle X_{1}, \ldots, X_{k}\right\rangle$, $\left[\operatorname{coef}\left(i_{1}, \ldots, i_{m}\right)\right](F)$ means the coefficient of $X_{i_{1}} \ldots X_{i_{m}}$ in $F(m \geq 1$ and 
$\left.1 \leq i_{1}, \ldots, i_{m} \leq k\right)$. If $V=\left\{h_{1}, \ldots, h_{r}\right\} \subset\{1, \ldots, m\}$ for some $h_{1}<\ldots<h_{r}$, then let $\left(i_{1}, \ldots, i_{m} \mid V\right)$ mean $\left(i_{h_{1}}, \ldots, i_{h_{r}}\right)$.

Let $a_{1}, \ldots, a_{k} \in \mathcal{A}$. A series $M_{\left(a_{1}, \ldots, a_{k}\right)} \in C_{\infty}\left\langle X_{1}, \ldots, X_{k}\right\rangle$ is called the moment generating series of the $k$-tuple $\left(a_{1}, \ldots, a_{k}\right)$ if

$$
\left[\operatorname{coef}\left(i_{1}, \ldots, i_{m}\right)\right]\left(M_{\left(a_{1}, \ldots, a_{k}\right)}\right)=\varphi\left(a_{i_{1}} \ldots a_{i_{m}}\right)
$$

for $m \geq 1$ and $1 \leq i_{1}, \ldots, i_{m} \leq k$ (see [9]).

Let $\mathcal{E}_{k}$ consist of the formal infinite sums

$$
T=\sum_{m, n=0}^{\infty} c_{i_{1}, \ldots, i_{m} ; j_{1}, \ldots, j_{n}} l_{i_{1}} \ldots l_{i_{m}} l_{j_{1}}^{*} \ldots l_{j_{n}}^{*},
$$

where $c_{i_{1}, \ldots, i_{m} ; j_{1}, \ldots, j_{n}} \in \mathbb{C}, i_{1}, \ldots, i_{m}, j_{1}, \ldots, j_{n} \in\{1, \ldots, k\}, l_{1}, \ldots, l_{k}$, $l_{1}^{*}, \ldots, l_{k}^{*}$ are non-commuting formal symbols (one can think of them as creation and annihilation operators), and there exists an $N \in \mathbb{N}$ such that $c_{i_{1}, \ldots, i_{m} ; j_{1}, \ldots, j_{n}}=0$ whenever $n>N$. Multiplication of the sums is governed by the computational rules $l_{i}^{*} l_{i}=1$ and $l_{i}^{*} l_{j}=0$ when $i \neq j$, while we add the elements of $\mathcal{E}_{k}$ in the usual way. The condition on the coefficients ensures that when multiplying two infinite sums we have to add only finitely many numbers. On $\mathcal{E}_{k}$ we define a normalized linear functional $\omega$ by $\omega(T)=c$, where $c$ is the constant term of the infinite sum $T$.

Let $a_{1}, \ldots, a_{k} \in \mathcal{A}$. A series $R_{\left(a_{1}, \ldots, a_{k}\right)} \in C_{\infty}\left\langle z_{1}, \ldots, z_{k}\right\rangle$ is called the $R$-series of the $k$-tuple $\left(a_{1}, \ldots, a_{k}\right)$ if for $T \in \mathcal{E}_{k}$,

$$
T=1+\sum_{m=0}^{\infty}\left[\operatorname{coef}\left(i_{1}, \ldots, i_{m}\right)\right]\left(R_{\left(a_{1}, \ldots, a_{k}\right)}\right) l_{i_{m}} l_{i_{m-1}} \ldots l_{i_{1}}
$$

and $\omega\left(l_{i_{1}}^{*} T \ldots l_{i_{l}}^{*} T\right)=\varphi\left(a_{i_{1}} \ldots a_{i_{l}}\right)$ for $l \geq 1$ and $1 \leq i_{1}, \ldots, i_{l} \leq k$ (see [6]).

It is important that for any $k$-tuple $\left(a_{1}, \ldots, a_{k}\right)$ the moment generating series $M_{a_{1}, \ldots, a_{k}}$ and the $R$-series $R_{a_{1}, \ldots, a_{k}}$ exist and are uniquely determined; moreover, according to the so-called moment-cumulant formula [9] we have

$$
\begin{array}{r}
{\left[\operatorname{coef}\left(i_{1}, \ldots, i_{m}\right)\right]\left(M_{\left(a_{1}, \ldots, a_{k}\right)}\right)} \\
=\sum_{\pi \in \mathrm{NC}(k)} \prod_{V \in \pi}\left[\operatorname{coef}\left(i_{1}, \ldots, i_{m} \mid V\right)\right]\left(R_{\left(a_{1}, \ldots, a_{k}\right)}\right),
\end{array}
$$

where the summation is over all non-crossing partitions of $\{1, \ldots, k\}$ and the product is over the blocks of the partition $\pi$. (The notions concerning non-crossing partitions are described at the beginning of Section 2.) One easily verifies that the moment-cumulant formula is invertible, that is, the coefficients of the $R$-series of any $k$-tuple can also be expressed uniquely by the coefficients of the moment generating series of the $k$-tuple (though that formula is somewhat more complicated; see e.g. [7]). 
Let $a_{1}, a_{2} \in \mathcal{A}$. The pair $\left(a_{1}, a_{2}\right)$ is an $R$-diagonal pair if the $R$-series of the pair is $R_{\left(a_{1}, a_{2}\right)}\left(z_{1}, z_{2}\right)=\sum_{k=1}^{\infty} \alpha_{k}\left(z_{1} z_{2}\right)^{k}+\sum_{k=1}^{\infty} \alpha_{k}\left(z_{2} z_{1}\right)^{k}$ for some sequence $\left\{\alpha_{k}\right\}_{k=1}^{\infty}$ of complex coefficients (see [7]).

An element $c$ of a $C^{*}$-probability space $(\mathcal{A}, \varphi)$ is called circular if the $R$-series of the pair $\left(c, c^{*}\right)$ is $R_{(c, c *)}\left(z_{1}, z_{2}\right)=z_{1} z_{2}+z_{2} z_{1}$.

2. Combinatorial background. For a finite set $S, \pi=\left\{V_{1}, \ldots, V_{p}\right\}$ is a partition of $S$ if the (non-empty) sets $V_{i}$ are disjoint and their union is $S$. The sets $V_{i}$ are called blocks of the partition. The number of blocks in a partition $\pi$ is denoted by $|\pi|$, the number of elements in a block $V_{i}$ is denoted by $\left|V_{i}\right|$. For a linearly ordered set $S$ we call the partition $\pi$ of $S$ crossing if in $\pi$ there are two blocks $V_{i} \neq V_{j}$ and elements $v_{1}, v_{2} \in V_{i}$, $w_{1}, w_{2} \in V_{j}$ such that $v_{1}<w_{1}<v_{2}<w_{2}$; otherwise $\pi$ is non-crossing. A partition $\pi$ of $S$ is called a pair-partition if every block of $\pi$ contains exactly two elements of $S$. The sets of all non-crossing partitions and of all non-crossing pair-partitions of $\{1, \ldots, k\}$ are denoted by $\mathrm{NC}(k)$ and $\mathrm{NCP}(k)$, respectively.

For further details of the theory of partitions see e.g. [1,4] and the references listed there.

Definition 2.1. Fix a function $i: \mathbb{N} \rightarrow\{1,2\}$ and $k, n \in \mathbb{N}$. Let $H_{i(1), \ldots, i(k)}=\left\{\pi \in \mathrm{NC}(k) \mid\right.$ for every $V=\left\{p_{1}, \ldots, p_{|V|}\right\} \in \pi$,

$|V|$ is even and $i\left(p_{j}\right) \neq i\left(p_{j+1}\right)$ for $\left.j=1, \ldots,|V|-1\right\}$

${ }_{(n)} \widetilde{H}_{i(1), \ldots, i(k)}=\{\nu \in \operatorname{NCP}(n k) \mid$ for every $V=\{p, q\} \in \nu$,

$$
i(] p / n[) \neq i(] q / n[)\}
$$

where $] x[$ means the smallest integer not smaller than $x$.

Moreover we call a partition $\pi$ of $\{1, \ldots, k\}$ an $n$-partition if every block of $\pi$ contains exactly $n$ elements. We denote the set of all non-crossing $n$-partitions of $\{1, \ldots, k\}$ by $\mathrm{NC}_{n}(k)$.

The cardinality of $\mathrm{NC}_{n}(\ln )$ is known to be the $l$ th generalized Catalan number of parameter $n$ given by

$$
{ }_{n} c_{l}=\frac{1}{(n-1) l+1}\left(\begin{array}{c}
n l \\
l
\end{array}\right) \quad(n, l \in \mathbb{N})
$$

(see e.g. [10]). Concerning the generalized Catalan numbers see also [3].

Lemma 2.2. Let $i: \mathbb{N} \rightarrow\{1,2\}$ be either

$$
i(j)=\left\{\begin{array}{ll}
1 & \text { if } j \text { is odd, } \\
2 & \text { if } j \text { is even, }
\end{array} \quad \text { or } \quad i(j)= \begin{cases}2 & \text { if } j \text { is odd, } \\
1 & \text { if } j \text { is even. }\end{cases}\right.
$$

Then the cardinality of ${ }_{(n)} \widetilde{H}_{i(1), \ldots, i(2 k)}$ equals that of $\mathrm{NC}_{n+1}(k(n+1))$. 
Proof. We define a bijection $T:{ }_{(n)} \widetilde{H}_{i(1), \ldots, i(2 k)} \rightarrow \mathrm{NC}_{n+1}(k(n+1))$.

First let $b$ be the function defined by

$$
\begin{gathered}
b:\{1, \ldots, 2 k n\} \rightarrow\{1, \ldots, k(n+1)\}, \\
s \mapsto b(s)= \begin{cases}\frac{1}{2} j(n+1)+l & \text { if } j \text { is even, } \\
\frac{1}{2} j+1(n+1) & \text { if } j \text { is odd, }\end{cases}
\end{gathered}
$$

where $j=[(s-1) / n]$ and $l=s-j n$; here $[x]$ stands for the integer part of $x$.

For any $\nu \in{ }_{(n)} \widetilde{H}_{i(1), \ldots, i(2 k)}$ let $T(\nu)$ be the partition of $\{1, \ldots, k(n+1)\}$ whose blocks are defined in the following way.

(1) If $\left\{s_{1}, s_{2}\right\} \in \nu$, then let $b\left(s_{1}\right)$ and $b\left(s_{2}\right)$ be in the same block of $T(\nu)$.

(2) If $r_{1}$ is in the same block as $r_{2}$, and $r_{2}$ is in the same block as $r_{3}$, then let $r_{1}$ and $r_{3}$ be also in the same block (transitivity).

Conditions (1) and (2) clearly define an equivalence relation, which gives a partition of $\{1, \ldots, k(n+1)\}$.

We show that $T(\nu) \in \mathrm{NC}_{n+1}(k(n+1))$ for every $\nu \in{ }_{(n)} \widetilde{H}_{i(1), \ldots, i(2 k)}$.

From the definition of ${ }_{(n)} \widetilde{H}_{i(1), \ldots, i(2 k)}$ for our special function $i$ : $\mathbb{N} \rightarrow\{1,2\}$ it follows that if $\left\{s_{1}, s_{2}\right\} \in \nu$, then one of $j_{1}=\left[\left(s_{1}-1\right) / n\right]$, $j_{2}=\left[\left(s_{2}-1\right) / n\right]$, say $j_{1}$, is odd, while the other is even. Then for every $s_{l} \in\{1, \ldots, 2 k n\}$ of the form $s_{l}=j_{1} n+l(l=1, \ldots, n)$ we have $b\left(s_{l}\right)=$ $\frac{1}{2}\left(j_{1}+1\right)(n+1)$. Take the block $V$ of $T(\nu)$ that contains $\frac{1}{2}\left(j_{1}+1\right)(n+1) \in$ $\{1, \ldots, k(n+1)\}$. Let the pair of $s_{l}$ in $\nu$ be $r_{l}$ (that is, let $r_{l} \in\{1, \ldots, 2 k n\}$ be (unique) such that $\left\{r_{l}, s_{l}\right\} \in \nu ; \nu$ is a pair-partition). As $\left[\left(s_{l}-1\right) / n\right]=j_{1}$ is odd, $j_{l}=\left[\left(r_{l}-1\right) / n\right]$ must be even. Let the "decomposition" of $r_{l}$ be $r_{l}=j_{l} n+h_{l}$. Then by $(1), b\left(r_{l}\right)=\frac{1}{2} j_{l}(n+1)+h_{l}$ is also in $V$ for every $l=1, \ldots, n$. This means that $V$ has at least $n+1$ elements, namely $b\left(s_{l}\right)$ (which is the same for every $l$ ) and $b\left(r_{l}\right)$ (which is different for different $l)$. Take any $x \in\{1, \ldots, 2 k n\}$ for which $b(x)=\frac{1}{2}\left(j_{1}+1\right)(n+1)$ or $b(x)=\frac{1}{2}\left(j_{l}+1\right)(n+1)+h_{l}(l=1, \ldots, n)$; the element $b(y)$ defined by the pair of $x$ is already in $V$ because of (1), so (2) cannot put any other element in $V$. This means that $V$ has exactly $n+1$ elements. By the same argument every block of $T(\nu)$ consists of exactly $n+1$ elements. It is also clear that in every block $V$ in $T(\nu)$ there is exactly one element of the form $\frac{1}{2} j(n+1)$ (call it $b$-odd), while the remaining ones are of the form of $\frac{1}{2} j(n+1)+l$ for some $l=1, \ldots, n$ (we will call them $b$-even).

Suppose that $T(\nu)$ is crossing for some $\nu \in{ }_{(n)} \widetilde{H}_{i(1), \ldots, i(2 k)}$. Then there are $V, U \in T(\nu)(V \neq U), v_{1}, v_{2} \in V, u_{1}, u_{2} \in U$ such that $v_{1}<u_{1}<v_{2}<u_{2}$. If one of $v_{1}$ and $v_{2}$ and one of $u_{1}$ and $u_{2}$ is $b$-odd, then there are blocks $\left\{s_{V}, r_{V}\right\}$ and $\left\{s_{U}, r_{U}\right\}$ of $\nu$ such that $b\left(s_{V}\right)=v_{1}, b\left(r_{V}\right)=v_{2}, b\left(s_{U}\right)=u_{1}$ and $b\left(r_{U}\right)=u_{2}$, where $s_{V}<s_{U}<r_{V}<r_{U}$. This means that $\nu$ is also crossing, 
which contradicts the fact that $\nu \in{ }_{(n)} \widetilde{H}_{i(1), \ldots, i(2 k)}$. If among $v_{1}, v_{2}, u_{1}, u_{2}$ there is only one $b$-odd element (say one of $v_{1}$ and $v_{2}$ ), then if the (unique) $b$-odd element $u_{0}$ of $U$ is smaller than $v_{1}$ or greater than $v_{2}$, then instead of $u_{2}$ we can take $u_{0}$ to get the "crossing elements" $v_{1}, v_{2}, u_{1}, u_{0}$ (if $u_{0}<v_{1}$ then $u_{0}<v_{1}<u_{1}<v_{2}$, if $u_{0}>v_{2}$ then $v_{1}<u_{1}<v_{2}<u_{0}$ ); while if $u_{0}$ is between $v_{1}$ and $v_{2}$, then $v_{1}, v_{2}, u_{0}, u_{2}$ will be good crossing elements containing two $b$-odd elements, leading to the same contradiction. If none of $v_{1}, v_{2}, u_{1}, u_{2}$ is $b$-odd, then if $u_{0}<v_{1}$ or $u_{0}>v_{2}$ then $v_{1}, v_{2}, u_{1}, u_{0}$, while if $v_{1}<u_{0}<v_{2}$ then $v_{1}, v_{2}, u_{0}, u_{2}$ are good crossing elements with one $b$-odd among them, leading again to the same contradiction. This finally shows that $T(\nu)$ is non-crossing for every $\nu \in{ }_{(n)} \widetilde{H}_{i(1), \ldots, i(2 k)}$, that is, $T(\nu) \in \mathrm{NC}_{n+1}(k(n+1))$ for every $\nu \in{ }_{(n)} \widetilde{H}_{i(1), i(2), \ldots, i(2 k)}$.

Next we show that $T$ is injective.

Let $\nu_{1}, \nu_{2} \in{ }_{(n)} \widetilde{H}_{i(1), \ldots, i(2 k)}$. We call $s \in\{1, \ldots, 2 k n\} j$-odd (resp. $j$-even) if in the decomposition $s=j n+l, j$ is odd (resp. even). The definition of ${ }_{(n)} \widetilde{H}_{i(1), \ldots, i(2 k)}$ shows that every block of $\nu \in{ }_{(n)} \widetilde{H}_{i(1), \ldots, i(2 k)}$ contains exactly one $j$-odd and one $j$-even element. So if $\nu_{1} \neq \nu_{2}$, then there is at least one $j$-even $s \in\{1, \ldots, 2 k n\}$ whose pairs in $\nu_{1}$ and in $\nu_{2}$ are different. Suppose that $\left\{s, r_{1}\right\} \in \nu_{1},\left\{s, r_{2}\right\} \in \nu_{2}$ and $r_{1}=j_{1} n+l_{1}, r_{2}=j_{2} n+l_{2}$, where $j_{1} \neq j_{2}$. In this case in $T\left(\nu_{1}\right), b(s)$ is in the same block as $b\left(r_{1}\right)=\frac{1}{2}\left(j_{1}+1\right)(n+1)$, while in $T\left(\nu_{2}\right), b(s)$ is in the same block as $b\left(r_{2}\right)=\frac{1}{2}\left(j_{2}+1\right)(n+1) \neq b\left(r_{1}\right)$. As $b\left(r_{1}\right)$ and $b\left(r_{2}\right)$ are both $b$-odd, they cannot be in the same block, so $T\left(\nu_{1}\right)$ and $T\left(\nu_{2}\right)$ are different.

Next we show that if there is no $j$-even $s \in\{1, \ldots, 2 k n\}$ such that in the decompositions of its pairs in $\nu_{1}$ and in $\nu_{2}, j_{1}=\left[\left(r_{1}-1\right) / n\right]$ and $j_{2}=\left[\left(r_{2}-1\right) / n\right]$ are different, then $\nu_{1}$ must be identical to $\nu_{2}$. In this case the set $\left\{s_{1}, \ldots, s_{n}\right\}$ of the pairs of the $n j$-odd elements of the form $r_{l}=j n+l$ $(l=1, \ldots, n)$ in $\nu_{1}$ is the same as that in $\nu_{2}$, for every $j=1, \ldots, 2 k-1$. But, as can easily be checked, there is only one pair-partition of the subset $\left\{s_{1}, \ldots, s_{n}, r_{1}, \ldots, r_{n}\right\} \subset\{1, \ldots, 2 k n\}$ which is non-crossing and in which every pair contains one $s_{l}$ and one $r_{\bar{l}}$, i.e. which can be a "subpartition" of a $\nu \in{ }_{(n)} \widetilde{H}_{i(1), \ldots, i(2 k)}$. This means that all the blocks of $\nu_{1}$ are the same as the blocks of $\nu_{2}$, proving the injectivity of $T$.

Finally we show that $T$ is surjective, by induction on $k$.

For $k=1$ the only element of $\mathrm{NC}_{n+1}(n+1)$ is $\pi=\{1, \ldots, n+1\}$. The partition $\nu=\{\{1,2 n\},\{2,2 n-1\}, \ldots,\{n, n+1\}\} \in{ }_{(n)} \widetilde{H}_{i(1), i(2)}$ clearly satisfies $T(\nu)=\pi$. Suppose that $T$ is surjective for $k$. We show that this implies the same for $k+1$. As $\pi \in \mathrm{NC}_{n+1}((k+1)(n+1))$ is non-crossing and every block of $\pi$ contains exactly $n+1$ elements, at least one block of $\pi$ contains the elements $\{p, p+1, \ldots, p+n\}$ for some $p \in\{1, \ldots, k(n+1)\}$. Among these elements there is exactly one of the form $j(n+1)$ for some 
$j=1, \ldots, k+1$. We denote it by $q$. Deleting the block $\{p, p+1, \ldots, p+n\}$ from $\pi$, we get a shorter partition $\widetilde{\pi} \in \mathrm{NC}_{n+1}(k(n+1))$. By assumption there exists a $\widetilde{\nu} \in{ }_{(n)} \widetilde{H}_{i(1), \ldots, i(2 k)}$ such that $\widetilde{\pi}=T(\widetilde{\nu})$. Consider the set $\{1, \ldots, 2 k n\}$ with its partition $\widetilde{\nu}$. Let

$$
s= \begin{cases}{\left[\frac{p}{n+1}\right] 2 n+\left(p-\left[\frac{p}{n+1}\right](n+1)\right)} & \text { if } q \neq p, \\ \frac{p}{n+1} 2 n-(n-1) & \text { if } q=p .\end{cases}
$$

This ensures that $b(s)=p$. Now put $2 n$ extra elements between $s-1$ and $s$, and renumber the whole set to get $\{1, \ldots, 2(k+1) n\}$. Let $\nu$ be the following pair-partition of this set. For the elements that came from our original set $\{1, \ldots, 2 k n\}$, their pairs in $\nu$ are defined by $\widetilde{\nu}$, while for the $2 n$ new elements we define their pairs to be $\left\{s_{h_{0}, j}, s_{h_{0}-j}\right\}$ for $j=1, \ldots, h_{0}$ and $\left\{s_{h_{0}, n-\tilde{j}, s_{h_{0}+1+\tilde{j}}}\right\}$ for $\widetilde{j}=0,1, \ldots, n-h_{0}-1$, where $h_{0}=q-p$, $s_{h}=\left[\frac{p+h}{n+1}\right] 2 n+\left(p+h-\left[\frac{p+h}{n+1}\right](n+1)\right) \quad$ for $h=0,1, \ldots, n, h \neq h_{0}$, and

$$
s_{h_{0}, l}=2\left(\frac{p+h_{0}}{n+1}-1\right) n+l \quad(l=1, \ldots, n) .
$$

As the given pair-partition of the $2 n$ new elements is non-crossing and so is $\widetilde{\nu} \in{ }_{(n)} \widetilde{H}_{i(1), \ldots, i(2 k)}, \nu$ is non-crossing. Moreover (as can be verified easily) the pair of every $j$-odd element of $\{1, \ldots, 2(k+1) n\}$ is $j$-even, which ensures that $\nu \in{ }_{(n)} \widetilde{H}_{i(1), \ldots, i(2(k+1))}$. In $T(\nu)$ the block that contains $q$ is clearly $\{p, p+1, \ldots, p+n\}$, while all the other blocks of $T(\nu)$ are defined by $\widetilde{\nu}$ (taken as a subpartition of $\nu$ ), so $T(\nu)=\pi$, which means that $T$ is surjective for $k+1$, and so for all $k$.

We conclude that $T:{ }_{(n)} \widetilde{H}_{i(1), \ldots, i(2 k)} \rightarrow \mathrm{NC}_{n+1}(k(n+1))$ is a bijection, which proves our statement.

Lemma 2.3. For any function $i: \mathbb{N} \rightarrow\{1,2\}$ and for all $k \in \mathbb{N}, n=$ $2,3,4, \ldots$,

$$
\#_{(n)} \widetilde{H}_{i(1), \ldots, i(k)}=\sum_{\pi \in H_{i(1), \ldots, i(k)}} \prod_{V \in \pi}{ }_{n-1} c_{|V| / 2} .
$$

Proof. For any $\nu \in{ }_{(n)} \widetilde{H}_{i(1), \ldots, i(k)}$ let $L(\nu)$ be the partition of $\{1, \ldots, k\}$ whose blocks are defined the following way.

(1) If $\left\{s_{1}, s_{2}\right\} \in \nu$, then let $\left.p_{1}=\right]\left(s_{1}-1\right) / n\left[\right.$ and $\left.p_{2}=\right]\left(s_{2}-1\right) / n[$ be in the same block of $L(\nu)$, where $] x[$ means the smallest integer no smaller than $x$.

(2) If $r_{1}$ is in the same block as $r_{2}$, and $r_{2}$ is in the same block as $r_{3}$, then let $r_{1}$ and $r_{3}$ be in the same block too (transitivity). 
Conditions (1) and (2) clearly define an equivalence relation, which gives a partition of $\{1, \ldots, k\}$.

Let $s_{l}(p)=(p-1) n+l(l=1, \ldots, n, p=1, \ldots, k)$. We show that for every $\nu \in{ }_{(n)} \widetilde{H}_{i(1), \ldots, i(k)}$ if $V=\left\{p_{1}, \ldots, p_{|V|}\right\}\left(p_{1}<\ldots<p_{|V|}\right)$ is a block of $L(\nu)$, then $\left\{s_{n}\left(p_{j}\right), s_{1}\left(p_{j+1}\right)\right\} \in \nu$ for $j=1, \ldots,|V-1|$, and $\left\{s_{n}\left(p_{|V|}\right), s_{1}\left(p_{1}\right)\right\} \in \nu$. As $\nu$ is a non-crossing pair-partition and as $i(] s_{1} / n[)$ $\neq i(] s_{2} / n[)$ for $\left\{s_{1}, s_{2}\right\} \in \nu$, the pair of $s_{1}(p)$ must be $s_{n}(q)$ for some $q=1, \ldots, k$, and the pair of $s_{n}(p)$ must be $s_{1}(\widetilde{q})$ for some $\widetilde{q}=1, \ldots, k$, for any $p=1, \ldots, k$. (Otherwise the number of elements $s$ between $s_{1}(p)$ and its pair for which $i(] s / n[)=1$ would differ from the number of those for which $i(] \widetilde{s} / n[)=2$, so $\nu$ could not belong to ${ }_{(n)} \widetilde{H}_{i(1), \ldots, i(k)}$.) Now, if the pair of $s_{n}\left(p_{j}\right)$ is not $s_{1}\left(p_{j+1}\right)$, then $p_{j}$ and $p_{j+1}$ could not be in the same block of $L(\nu)$ (again because $\nu$ is non-crossing).

The pair of $s_{n}\left(p_{|V|}\right)$ cannot be $s_{1}(p)$ for $p>p_{|V|}$ or $p<p_{|V|}$, because in this case $p_{|V|}$ would not be the largest, or $p_{1}$ would not be the smallest element in $V$. But if the pair of $s_{n}\left(p_{|V|}\right)$ is $s_{1}(p)$ for some $p_{1}<p<p_{|V|}$, then - as $\nu$ is non-crossing - $p_{1}$ and $p_{|V|}$ could not be in the same block of $L(\nu)$. It follows that the pair of $s_{n}\left(p_{|V|}\right)$ is necessarily $s_{1}\left(p_{1}\right)$.

Next we show that $L(\nu)$ is non-crossing for every $\nu \in{ }_{(n)} \widetilde{H}_{i(1), \ldots, i(k)}$. Suppose that some $L(\nu)$ is crossing. Then there are $V, U \in L(\nu)(V \neq U)$, $v_{1}, v_{2} \in V, u_{1}, u_{2} \in U$ such that $v_{1}<u_{1}<v_{2}<u_{2}$. Let $\widetilde{v}_{1}$ be the largest element of $V$ which is still smaller than $u_{1}$; let $\widetilde{v}_{2}$ be the smallest element of $V$ which is still greater than $u_{1}$; let $\widetilde{u}_{1}$ be the largest element of $U$ which is still smaller than $\widetilde{v}_{2}$, and let $\widetilde{u}_{2}$ be the smallest element of $U$ which is still greater than $\widetilde{v}_{2}$ (such elements clearly exist). This implies that there is no element of $V$ between $\widetilde{v}_{1}$ and $\widetilde{v}_{2}$ nor an element of $U$ between $\widetilde{u}_{1}$ and $\widetilde{u}_{2}$, and still $\widetilde{v}_{1}<\widetilde{u}_{1}<\widetilde{v}_{2}<\widetilde{u}_{2}$. But, as we saw above, in this case $\left\{s_{n}\left(\widetilde{v}_{1}\right), s_{1}\left(\widetilde{v}_{2}\right)\right\} \in \nu$ and $\left\{s_{n}\left(\widetilde{u}_{1}\right), s_{1}\left(\widetilde{u}_{2}\right)\right\} \in \nu$. As $s_{n}\left(\widetilde{v}_{1}\right)<s_{n}\left(\widetilde{u}_{1}\right)<s_{1}\left(\widetilde{v}_{2}\right)<s_{1}\left(\widetilde{u}_{2}\right), \nu$ would also be crossing, a contradiction.

Next we show that for every $\nu \in{ }_{(n)} \widetilde{H}_{i(1), \ldots, i(k)}$ and for every block $V=$ $\left\{p_{1}, \ldots, p_{|V|}\right\}\left(p_{1}<\ldots<p_{|V|}\right)$ of $L(\nu),|V|$ is even and $i\left(p_{j}\right) \neq i\left(p_{j+1}\right)$ $(j=1, \ldots,|V|-1)$. We saw above that $\left\{s_{n}\left(p_{j}\right), s_{1}\left(p_{j+1}\right)\right\} \in \nu$, which yields

$$
i\left(p_{j}\right)=i(\rfloor \frac{s_{n}\left(p_{j}\right)-1}{n}\lceil) \neq i(\rfloor \frac{s_{1}\left(p_{j+1}\right)-1}{n}\lceil)=i\left(p_{j+1}\right)
$$

while $\left\{s_{n}\left(p_{|V|}\right), s_{1}\left(p_{1}\right)\right\} \in \nu$ gives

$$
i\left(p_{1}\right)=i(\rceil \frac{s_{1}\left(p_{1}\right)-1}{n}\lceil) \neq i(\rceil \frac{s_{n}\left(p_{|V|}\right)-1}{n}\lceil)=i\left(p_{j+1}\right),
$$

which proves our statement. This means that $L(\nu) \in H_{i(1), \ldots, i(k)}$ for every $\nu \in(n) \widetilde{H}_{i(1), \ldots, i(k)}$. 
We show that $L$ is surjective, that is, for any $\pi \in H_{i(1), \ldots, i(k)}$ there exist (several) $\nu \in{ }_{(n)} \widetilde{H}_{i(1), \ldots, i(k)}$ such that $L(\nu)=\pi$. Let $\pi=\left\{V_{1}, \ldots, V_{|\pi|}\right\}$, and let $V_{j}=\left\{p_{1}^{(j)}, \ldots, p_{\left|V_{j}\right|}^{(j)}\right\}\left(p_{1}^{(j)}<\ldots<p_{\left|V_{j}\right|}^{(j)}, j=1, \ldots,|\pi|\right)$. Let $\nu$ be the pair-partition of $\{1, \ldots, k n\}$ which consists of the blocks $\left\{s_{1}\left(p_{1}^{(j)}\right), s_{n}\left(p_{\left|V_{j}\right|}^{(j)}\right)\right\}$, $\left\{s_{n}\left(p_{m}^{(j)}\right), s_{1}\left(p_{m+1}^{(j)}\right)\right\}$ and $\left\{s_{l}\left(p_{2 t-1}^{(j)}\right), s_{n+1-l}\left(p_{2 t}^{(j)}\right)\right\}$, where $j=1, \ldots,|\pi|$, $m=1, \ldots,\left|V_{j}\right|-1, t=1, \ldots,\left|V_{j}\right| / 2$ and $l=2, \ldots, n-1$. Clearly $\nu \in$ ${ }_{(n)} \widetilde{H}_{i(1), \ldots, i(k)}$ and $L(\nu)=\pi$.

Note that the blocks $\left\{s_{1}\left(p_{1}^{(j)}\right), s_{n}\left(p_{\left|V_{j}\right|}^{(j)}\right)\right\}$ and $\left\{s_{n}\left(p_{m}^{(j)}\right), s_{1}\left(p_{m+1}^{(j)}\right)\right\}(m=$ $\left.1, \ldots,\left|V_{j}\right|-1, j=1, \ldots,|\pi|\right)$ of $\nu$ already define all the blocks of $L(\nu)$; and the fact that $\nu$ is non-crossing ensures that the rest of the blocks of $\nu$ do not define any other elements in any of the blocks of $L(\nu)$. This means that on the elements $\left\{s_{l}\left(p_{t}^{(j)}\right)\right\}$ (where $l=2, \ldots, n-1, t=1, \ldots,\left|V_{j}\right|$ and $j=1, \ldots,|\pi|)$ any other non-crossing pair-partition $\widetilde{\nu}$-given in such a way that if $\left\{s_{1}, s_{2}\right\} \in \widetilde{\nu}$, then $i(]\left(s_{1}-1\right) / n[) \neq i(]\left(s_{2}-1\right) / n[)$-satisfies $L(\widetilde{\nu})=\pi$. If we take into account that for any $\pi \in H_{i(1), \ldots, i(k)}$ and for every block $V_{j}=\left\{p_{1}^{(j)}, \ldots, p_{\left|V_{j}\right|}^{(j)}\right\}$ of $\pi$ we have $i\left(p_{m}^{(j)}\right) \neq i\left(p_{m+1}^{(j)}\right)$, and that the subpartitions of $\widetilde{\nu}$ that define different blocks of $\pi=L(\widetilde{\nu})$ are independent of each other, Lemma 2.2 completes the proof.

\section{Results}

DeFinition 3.1. Let $(\mathcal{A}, \varphi)$ be a non-commutative probability space, and let $b_{1}, b_{2} \in \mathcal{A}$. We call $\left(b_{1}, b_{2}\right)$ a circular pair if its $R$-series is $R_{\left(b_{1}, b_{2}\right)}\left(z_{1}, z_{2}\right)=$ $z_{1} z_{2}+z_{2} z_{1}$.

TheOREM 3.2. Let $(\mathcal{A}, \varphi)$ be a non-commutative probability space, $b_{1}, b_{2}$ $\in \mathcal{A}$, and let $\left(b_{1}, b_{2}\right)$ be a circular pair. Then the pair $\left(b_{1}^{n}, b_{2}^{n}\right)$ is $R$-diagonal for every $n=2,3, \ldots$ Moreover, the $R$-series of $\left(b_{1}^{n}, b_{2}^{n}\right)$ is given by

$$
R_{\left(b_{1}^{n}, b_{2}^{n}\right)}\left(z_{1}, z_{2}\right)=\sum_{k=1}^{\infty}{ }_{n-1} c_{k}\left(z_{1} z_{2}\right)^{k}+\sum_{k=1}^{\infty}{ }_{n-1} c_{k}\left(z_{2} z_{1}\right)^{k},
$$

where ${ }_{n-1} c_{k}=\frac{1}{(n-2) k+1}\left(\begin{array}{c}(n-1) k \\ k\end{array}\right)$ is the $k$ th generalized Catalan number of parameter $n-1$.

Proof. We use the notations of Definition 2.1.

For every $R$-diagonal pair $\left(a_{1}, a_{2}\right)$ its $R$-series is of the form $R_{\left(a_{1}, a_{2}\right)}\left(z_{1}, z_{2}\right)$ $=\sum_{k=1}^{\infty} \alpha_{k}\left(z_{1} z_{2}\right)^{k}+\sum_{k=1}^{\infty} \alpha_{k}\left(z_{2} z_{1}\right)^{k}$ for some sequence $\left\{\alpha_{k}\right\}_{k=1}^{\infty}$ of complex coefficients. According to the moment-cumulant formula (1.1) this implies

$$
[\operatorname{coef}(i(1), \ldots, i(m))]\left(M_{\left(a_{1}, a_{2}\right)}\right)=\sum_{\pi \in H_{i(1), \ldots, i(m)}} \prod_{V \in \pi} \alpha_{|V| / 2}
$$

for $m \geq 1$ and for every function $i: \mathbb{N} \rightarrow\{1,2\}$. 
From now on let $a_{1}=b_{1}^{n}, a_{2}=b_{2}^{n}$ for some fixed $n \in\{2,3, \ldots\}$. In this case

$$
\begin{aligned}
& {[\operatorname{coef}(i(1), \ldots, i(m))]\left(M_{\left(a_{1}, a_{2}\right)}\right)=\varphi\left(a_{i(1)} \ldots a_{i(m)}\right)} \\
& \quad=\varphi\left(b_{i(1)}^{n} \ldots b_{i(m)}^{n}\right)=\varphi\left(b_{i(1)} \ldots b_{i(1)} b_{i(2)} \ldots b_{i(2)} \ldots b_{i(m)} \ldots b_{i(m)}\right) \\
& \quad \stackrel{(1)}{=} \sum_{\pi \in H_{i(1), \ldots, i(1), i(2), \ldots, i(2), \ldots, i(m), \ldots, i(m)}} \prod_{V \in \pi} \alpha_{|V| / 2} \\
& \quad \stackrel{(2)}{=} \sum_{\nu \in(n)} \prod_{\widetilde{H}_{i(1), \ldots, i(m)}} \prod_{V \in \nu} 1=\# \widetilde{H}_{(n)} \widetilde{H}_{i(1), \ldots, i(m)} \\
& \quad \sum_{\pi \in H_{i(1), \ldots, i(m)}} \prod_{V \in \pi} \prod_{n-1} c_{|V| / 2} .
\end{aligned}
$$

Here the first equality comes from the definition of the moment generating series; (1) holds because $\left(b_{1}, b_{2}\right)$ is a circular pair, which means that it is also $R$-diagonal for some sequence $\left\{\alpha_{k}\right\}_{k=1}^{\infty} ;(2)$ can be obtained by taking into account that in the $R$-series of circular pairs the only coefficients that do not vanish are those of $z_{1} z_{2}$ and of $z_{2} z_{1}$, which are both 1 ; and (3) follows from Lemma 2.3. This means that the moment generating series of $\left(b_{1}^{n}, b_{2}^{n}\right)$ is given by formula (3.1) for every $n \geq 2(m \geq 1, i: \mathbb{N} \rightarrow\{1,2\})$ for the sequence $\left\{{ }_{n-1} c_{k}\right\}_{k=1}^{\infty}$. Since the moment-cumulant formula is invertible, this completes the proof.

\section{Theorem 3.2 immediately yields}

COROllary 3.3. For a circular element $c$ of a $C^{*}$-probability space $(\mathcal{A}, \varphi)$ the pair $\left(c^{n},\left(c^{*}\right)^{n}\right)$ is $R$-diagonal for every $n \geq 1$.

REMARK 3.4. If $n=2$, then all the coefficients $\alpha_{k}$ are 1 , that is,

$$
R_{\left(b_{1}^{2}, b_{2}^{2}\right)}\left(z_{1}, z_{2}\right)=\sum_{k=1}^{\infty}\left(z_{1} z_{2}\right)^{k}+\sum_{k=1}^{\infty}\left(z_{2} z_{1}\right)^{k} .
$$

If $n=3$, then the coefficients $\left\{\alpha_{k}\right\}_{k=1}^{\infty}$ coincide with the sequence $\left\{c_{k}\right\}_{k=1}^{\infty}$ of Catalan numbers, so

$$
R_{\left(b_{1}^{3}, b_{2}^{3}\right)}\left(z_{1}, z_{2}\right)=\sum_{k=1}^{\infty} c_{k}\left(z_{1} z_{2}\right)^{k}+\sum_{k=1}^{\infty} c_{k}\left(z_{2} z_{1}\right)^{k},
$$

where $c_{k}={ }_{2} c_{k}=\frac{1}{k+1}\left(\begin{array}{c}2 k \\ k\end{array}\right)$.

REMARK 3.5. From the proof of Theorem 3.2 the following facts are obvious for any $R$-diagonal pair $\left(a_{1}, a_{2}\right)$ :

(a) $\varphi\left(a_{i(1)} \ldots a_{i(m)}\right)=0$ whenever $m$ is odd, 
(b) $\varphi\left(a_{i(1)} \ldots a_{i(m)}\right)=0$ whenever

$\#\{j \in\{1, \ldots, m\}: i(j)=1\} \neq \#\{j \in\{1, \ldots, m\}: i(j)=2\}$,

(c) $\varphi\left(\bar{a}_{i(1)} \ldots \bar{a}_{i(m)}\right)=\varphi\left(a_{i(1)} \ldots a_{i(m)}\right)$ for $m \geq 1$ and for every $i: \mathbb{N} \rightarrow$ $\{1,2\}$, where $\bar{a}_{1}=a_{2}$ and $\bar{a}_{2}=a_{1}$.

\section{References}

[1] G. E. Andrews, The Theory of Partitions, Addison-Wesley, Reading, 1976.

[2] F. Hiai and D. Petz, The semi-circle law, free random variables and entropy, to be published.

[3] P. Hilton and J. Pedersen, Catalan numbers, their generalization, and their uses, Math. Intelligencer 13 (1991), no. 2, 64-75.

[4] G. Kreweras, Sur les partitions non-croisées d'un cycle, Discrete Math. 1 (1972), 333-350.

[5] F. Larsen, On the powers of $R$-diagonal elements, J. Operator Theory (to appear).

[6] A. Nica, R-transform of free joint distributions and non-crossing partitions, J. Funct. Anal. 135, (1996), 271-296.

[7] A. Nica and R. Speicher, R-diagonal pairs - a common approach to Haar unitaries and circular elements, in: Free Probability Theory, D. V. Voiculescu (ed.), Fields Inst. Comm. 12, Amer. Math. Soc., 1997, 149-188.

[8] A. Nica, D. Shlyakhtenko and R. Speicher, Some minimization problems for the free analogue of Fisher information, Adv. Math. 141 (1999), 282-321.

[9] R. Speicher, Multiplicative functions on the lattice of non-crossing partitions and free convolution, Math. Ann. 298 (1994), 611-628.

[10] - Combinatorial theory of the free product with amalgamation and operator-valued free probability theory, Mem. Amer. Math. Soc. 627 (1998).

[11] D. Voiculescu, Circular and semicircular systems and free product factors, in: Operator Algebras, Unitary Representations, Enveloping Algebras, and Invariant Theory, A. Connes et al. (eds.), Birkhäuser, 1990, 45-60.

[12] D. V. Voiculescu, K. J. Dykema and A. Nica, Free Random Variables, CRM Monograph Ser. 1, Amer. Math. Soc., 1992.

Mathematical Institute of the Hungarian Academy of Sciences

Reáltanoda u. 13-15

H-1053 Budapest, Hungary

E-mail: oravecz@math-inst.hu

Received June 28, 1999

Revised version September 19, 2000 